\title{
Dynamic Simulation Analysis of Off-axis Sensitivity in a Laterally-driven MEMS Inertia Switch
}

\author{
Qihuan Zhang ${ }^{1, a}$, Zhuoqing Yang ${ }^{2, b_{\star}}$ and Guifu Ding ${ }^{3, c}$ \\ 1, 2, 3 National Key Laboratory of Science and Technology on Micro/Nano Fabrication, \\ School of Electronic Information and Electrical Engineering, \\ Shanghai Jiao Tong University, Shanghai, 200240, P. R. China \\ a zhangqihuan@sjtu.edu.cn, ${ }^{\text {b }}$ yzhuoqing@sjtu.edu.cn, ${ }^{c}$ gfding@sjtu.edu.cn
}

Keywords: MEMS, inertial micro-switch, off-axis sensitivity, contact enhancement

Abstract. A laterally-driven MEMS inertia switch was designed, and its off-axis sensitivity was analyzed and evaluated. The dynamic response process was simulated by ANSYS finite-element model, which indicated that in the designed inertia micro-switch, the distribution of serpentine spring symmetry, the proof mass weight and the whole system stiffness have significant effect on the off-axis sensitivity.

\section{Introduction}

Inertial micro-switch based on Micro-electromechanical Systems (MEMS), as a typical kind of acceleration sensor controlled by threshold acceleration, is widely used in many industrial applications such as automobiles and toys [1,2]. Especially, internet of things (IOT) system has grown rapidly in recent years, so the inertia micro-switch with the advantages of small size, lower costs and large volume production has attracted interest $[3,4]$. It can be used for remote detecting vibration shock and then giving corresponding triggering signals, not only as a threshold sensor but will also an actuator.

The working principle of an inertia switch is that the movable electrode (usually a suspended proof mass) contacts a fixed electrode when the switch is accelerated up to the threshold level, connecting to form an electric path. The inertial micro-switch will be inevitably subjected to some acceleration from different directions because of the complicated working environment. If an inertia micro-switch is subjected to a acceleration in off-axis direction, the suspended proof mass will impact to constraint structure, lowering the shock-stability and shorten service life. Although high-g inertial micro-switches have been reported [5-7], few researches focus on how to lower off-axis sensitivity. With regards to this, in the present work, finite element software ANSYS was used to simulated and evaluated off-axis sensitivity in a laterally-driven MEMS inertia switch to improving the shock-stability.

\section{Structure design}

A laterally-driven inertial micro-switch which can effectively lower the off-axis sensitivity and enhance the single-axial sensitivity is designed, as shown in Fig. 1. The device consists of three main parts: the double-layer cantilever beam as the movable electrode, which attaches to the proof mass suspended by double-layer serpentine spring, as shown in Fig. 1(b); one block located at the front of proof mass as the statical electrode and the compact constraint structures consisting of constraint sleeve with holes and reverse constraint blocks. The thickness of proof mass is much larger than spring thickness, which ensures that the whole system has higher sensitivity in sensing-axis direction. The sensitive direction of the device is parallel to the substrate plane, when sufficient acceleration is applied in the sensitive direction, the two-double cantilever beam which attaches to the proof mass will move rapidly toward and impact the statical electrode. And a contact enhancement between two electrodes could be realized in this system, attributed to the elastic deformation of the double-layer cantilever beam. Simultaneously, if the proof mass was also subjected to a small acceleration 
disturbance in off-axis direction, the proof mass will move in corresponding direction. The off-axis sensitivity of inertial micro-switch is the ratio value of the maximum displacement of the proof mass in the off-axis direction and in the sensitive direction. In the design, the off-axis sensitivity can be measured by off-axis displacement because the axis displacement is the gap between cantilever beam and stationary electrode when the applied acceleration is large. As shown in Fig.1(a), the constraint sleeve is introduced to limit random movement and to protect the device from damaging in the vertical $\mathrm{z}$ direction and in the horizontal y direction when the inertial micro-switch is subjected from complicated shocks. Fig.1(c) presents that the reverse constraint blocks are used to improve shock-resistibility in the reverse sensitive direction.

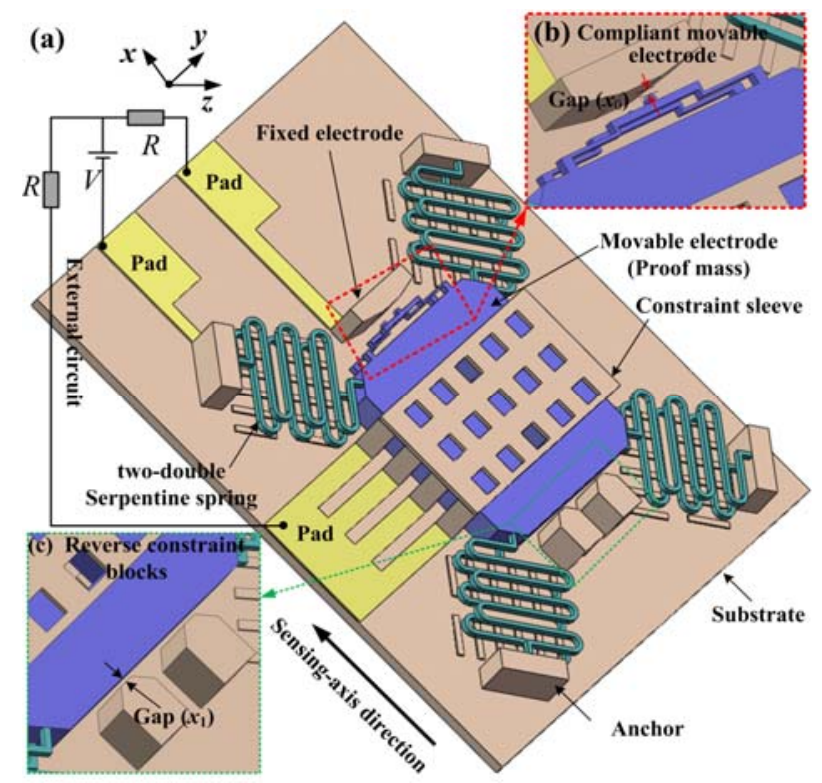

Fig. 1. The structure diagram of inertial micro-switch. (a) The 3D view of structure. (b) The enlarged view of movable electrode (c) The enlarged view of gap between proof mass and reverse blocks.

\section{Simulation}

The commercial ANSYS finite-element (FE) software is used to simulate the dynamic contact process, which can provide us a convenient and precise method for evaluating the off-axis sensitivity when the proof mass is subjected to a small acceleration disturbance in off-axis direction. Fig. 2 presents the FE model for the whole inertia switch and the close-up of contact pair between static electrode and compliant movable electrode. All Degrees-of-Freedoms (DOFs) of the end sections of spring, static electrode and blocks are constrained to zero. SOLID 185, CONTA174 and TARGE 170 and SWEEP method are used to mesh the model. Experimentally, the basic material of the inertia switch is electroplated nickel and its properties are as follows: Poisson's ratio is 0.3, Young's modulus is $150 \mathrm{GPa}$ and density is $8.96 \mathrm{~g} \cdot \mathrm{cm}^{-3}$. 


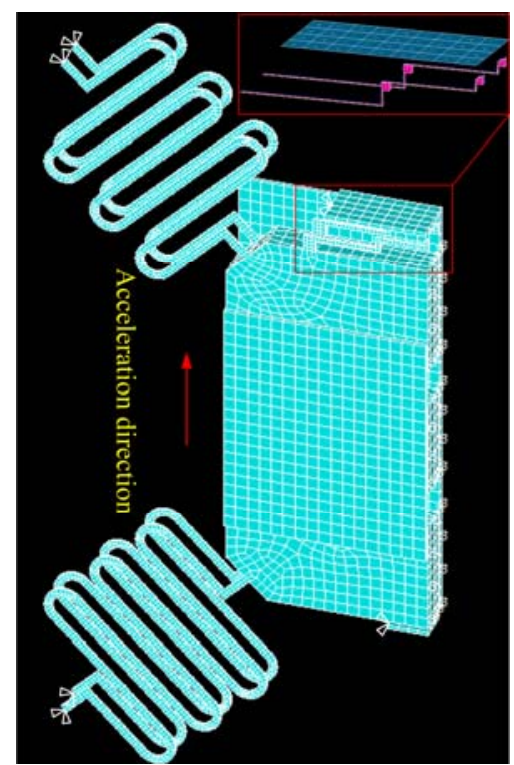

Fig. 2. FE model of designed inertial micro-switch and its contact pair for dynamic simulation analysis.

Generally, when the proof mass is subjected to a small acceleration disturbance in off-axis direction, the proof mass will move in corresponding direction. The larger the displacement is, the greater the off-axis sensitivity is. The greater off-axis sensitivity can lead to fierce collision with constraint sleeve, and even rotate in in the $x O z$ plane, lowering the shock-stability of inertia micro-switch. Experimentally, the off-axis sensitivity changes with the distribution of serpentine spring symmetry, stiffness of the whole inertia micro-switch system and different weight of proof mass.

(a)

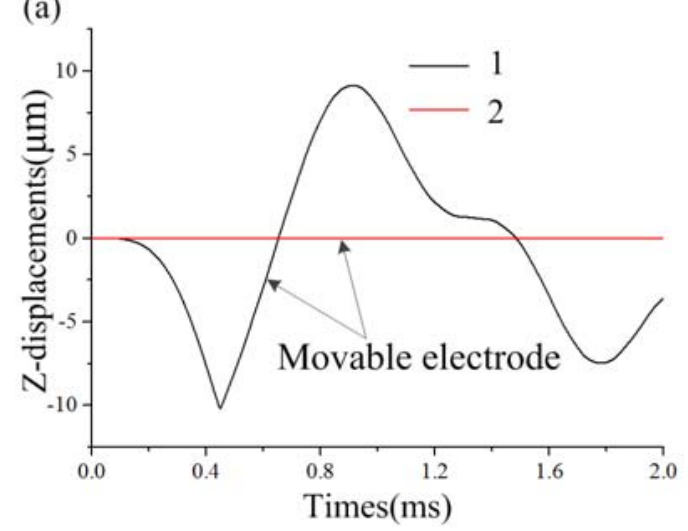

(b)

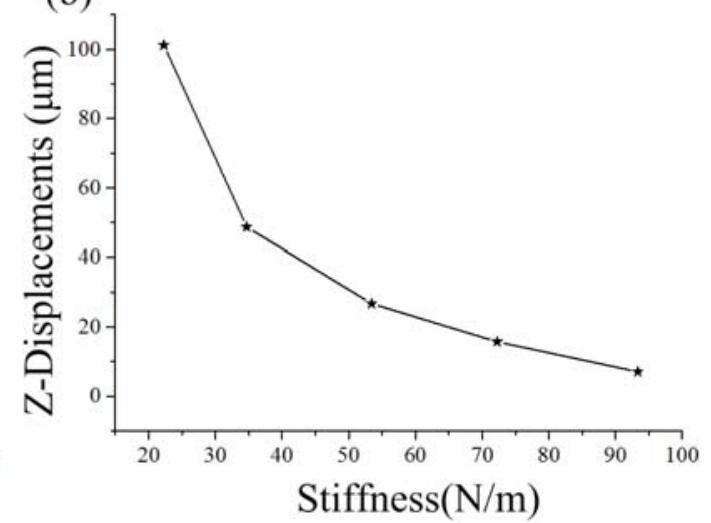

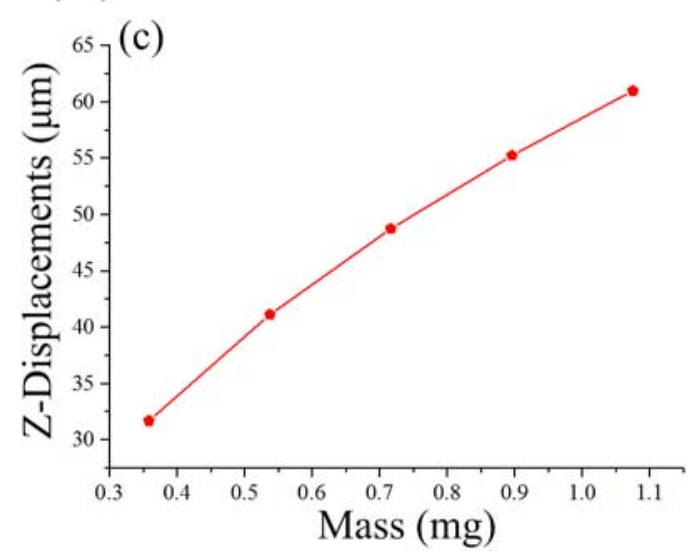

Fig. 3. The off-axis sensitivity changes with the distribution of serpentine spring symmetry (a), different weight of proof mass (b), and stiffness of the whole system (c).

Fig.3 (a) shows the $z$-displacement of proof mass changes with the distribution of serpentine spring symmetry. The unsymmetrical distribution of a layer serpentine spring has large off-sensitivity 
with about $10 \mu \mathrm{m} z$-displacement. This is because the proof mass does not move only in the sensitive direction, but will also rotate in the $x O z$ plane due to the centroid deviation between proof mass and serpentine spring. The double-layer serpentine spring is symmetrical distribution, and its $\mathrm{z}$-displacement is smaller than $0.1 \mu \mathrm{m}$, effectively lowering the off-axis sensitivity. Thus, the symmetrical distributions of double-layer serpentine spring can effectively lowering the off-axis sensitivity.

Fig.3 (b) presents the $z$-displacement of proof mass changes with stiffness of the whole inertia micro-switch system, when the inertia micro-switch is applied a $170 \mathrm{~g}$ amplitude acceleration in sensitivity direction and a $17 \mathrm{~g}$ amplitude acceleration in $z$ direction. When the stiffness is $22 \mathrm{~N} / \mathrm{m}$, $35 \mathrm{~N} / \mathrm{m}, 53 \mathrm{~N} / \mathrm{m}, 72 \mathrm{~N} / \mathrm{m}$ and $93 \mathrm{~N} / \mathrm{m}$, the maximum displacement of proof mass are $101 \mu \mathrm{m}, 48 \mu \mathrm{m}$, $27 \mu \mathrm{m}, 16 \mu \mathrm{m}$, and $7 \mu \mathrm{m}$, respectively. And in this simulation, the simulation model does not include constraint structures because the aim is off-axis sensitivity of inertia micro-switch system. It is clearly to see that as the stiffness get stronger, the maximum z-displacement of proof mass will be smaller, and the off-axis sensitivity of inertia micro-switch will get lower.

Fig.3 (c) presents the z-displacement of proof mass changes with weight of proof mass, when the inertia micro-switch is applied a $170 \mathrm{~g}$ amplitude acceleration in sensitivity direction and a $17 \mathrm{~g}$ amplitude acceleration in $z$ direction. The mass $\mathrm{m}$ changes from $0.36 \mathrm{mg}$ to $1.08 \mathrm{mg}$ (i.e. $0.36 \mathrm{mg}$, $0.54 \mathrm{mg}, 0.72 \mathrm{mg}, 0.90 \mathrm{mg}, 1.08 \mathrm{mg}$ ), the maximum displacement of proof mass in $z$ direction are $32 \mu \mathrm{m}$, $41 \mu \mathrm{m}, 49 \mu \mathrm{m}, 55 \mu \mathrm{m}$, and $61 \mu \mathrm{m}$, respectively. In the same way, the simulation model does not include constraint structures. And the plot implied the off-axis sensitivity rises with the increase of weight of proof mass.

\section{Conclusions}

A laterally-driven MEMS inertia switch was designed, and the off-axis sensitivity of inertia switch was simulated and evaluated by the finite-element software ANSYS. Simulation results reveal that the symmetrical distribution of double-layer serpentine spring can effectively lowering the off-axis sensitivity. As the stiffness get stronger, the off-axis sensitivity of inertia micro-switch will get lower and the off-axis sensitivity rises with the increase of weight of proof mass.

\section{Acknowledgements}

This work was financially supported by the Hi-Tech Research and Development Program of China (2015AA042701) and the National Key Laboratory of Micro/Nano Fabrication Technology Foundation (9140C790403150C79332).

\section{References}

[1] M.R. Whitley, M. Kranz and R. Kesmodel: SPIE 5717 (2004) 185-194.

[2] Y. Gerson and D. Schreiber: J. Micromech. Microeng. 24025008

[3] T. Matsunaga and M. Esashi: Sens. Actuators A 100 (2002) 10-17.

[4] A. Ongkodjojo: Phys. Conf. Ser. 34 1044-1052

[5] H. G. Cai, G. F. Ding and Z. Q. Yang: J. Micromech. Microeng. vol. 18, 115033(10pp), 2008

[6] Z.Q. Yang, B. Zhu and W.G .Chen: J. Micromech. Microeng. 22045006

[7] W.G .Chen, Z.Q. Yang and Y.L. Wang: Sens. Actuators A 216 7-18. 